\title{
An Electromagnetic Structure Simulator for Innovative Engineering Technology
}

\author{
Fabio Urbani, Juan R. Iglesias, Iram Lee, Jose F. Treviño \\ the University of Texas at Brownsville \\ furbani@utb.edu
}

Introduction

The great interest in microwave frequencies arises for a variety of reasons. Basic among these is the ever-increasing need for more radio-frequency-spectrum space and the rather unique uses to which microwave frequencies can be applied. In more recent years microwave frequencies have also come into widespread use in communication links, generally referred to as microwave links. The applications of microwaves and microwave technology extend much further, into a variety of areas of basic and applied research, and include a number of diverse practical devices, such as microwave ovens, sensors, and antennas.

Most of the microwave components require a very careful design phase in which the behavior of the electromagnetic field within the entire structure must be predicted in order to avoid undesired effects due to tool tolerances, material imperfections and packaging.

In order to understand how the electromagnetic field behaves within a given electromagnetic structure, a computational aid is indispensable to help engineers understand the field distribution. Even though a number of commercial analysis packages are currently available, they are rather expensive, and the source code is not available. The latter prevents students from understanding the logic behind the simulation.

In this paper we describe an ongoing project being developed at the University of Texas at Brownsville (UTB) that intends to overcome these limitations by implementing, based on a consolidated frequency domain technique, a novel electromagnetic structure simulator using a pre-existent 94-node Beowulf machine.

The merit and innovation of this project is how it integrates disperse tools and techniques into a single coherent framework that can be utilized with minimal effort and high confidence by the community interested in the research, teaching, learning and diffusion of electromagnetic wave simulation and analysis.

The technique used by the simulator is the Finite Elements Method (FEM). Because of its unique features, the FEM is becoming the workhorse for electromagnetic modeling and simulations. Many research and development codes that are now available in universities and industries, have demonstrated the utility and capability of the method. 
In terms of educational value, this project aims to self-train the students involved and to provide tutoring that leads to the formation of self-sufficient team equipped to manage and develop new features with the minimum support of faculty members. In this respect, the project represents a new frontier for Engineering Technology students at UTB.

General Analysis Requirements

The technique used by the simulator is the Finite Elements Method (FEM). Although other numerical methods like the finite difference method (FDM) and the method of moments (MOM) are conceptually simpler and easier to program, FEM is a more powerful and versatile numerical technique for handling problems involving complex geometries and non-homogeneous media. The systematic generality of the method makes possible to construct general purpose computer programs to solve a wide range of problems including open-geometry problems with the aid of hybrid methods like absorbing boundary conditions (ABC). Therefore, programs developed for a particular discipline have been applied successfully to solve problems in different areas with little or no modification ${ }^{1}$.

From a practical point of view the simulator will take as input a set of physical parameters and will return the spatial distribution of the electromagnetic field within the given structure. The envisioned simulator uses optimizing routines aimed to modify the physical and geometrical parameters of the given structure in order to modify the electromagnetic field distribution to match the initial requirements. This simulator contains three different optimizing methods: the Monte Carlo Method, the Genetic Algorithm Method, and the Tabu Search Method.

In order to approach any FEM problem, four basic steps must be followed:

1. Discretize the domain in which the solution has to be found using a finite number of nodes and elements (either triangular or quadrilateral);

2. Derive the governing equations for a single element;

3. Assemble all the elements in the solution domain;

4. Solve the system of equations obtained using numerical techniques like the Iteration Method or the Band Matrix Method. The former is faster and is usually preferred for a large number of nodes;

Differential equations can be applied to general electromagnetic problems and it is often convenient to work with potential as the dependent variable. This is because potential is a scalar and because the specified boundary conditions are often given in terms of potentials. In this light, the Laplace equation is a fundamental equation in more than one field of science $^{2}$.

The current goal is to find the solution of the Laplace equation for the potential into a twodimensional domain. Once this has been achieved, the objective will evolve to simulate structures that are more complicated. The algorithm to obtain the potential in the 2-D domain has been set up and is being implemented. 


\section{System Architecture}

Figure 1 shows the system architecture that is pursued in the long term. The aim is to build software that can use a number of parallel platforms including clusters, Beowulf, SMP and SISD systems. This property is especially important because of the computational power required to explore all the points of an electromagnetic field. Well known libraries for parallel programming are used in the project to handle the hardware like MPI. The access to the hardware is encapsulated through a hardware application program interface (API). This layer is important to maintain the portability of the project among several platforms.
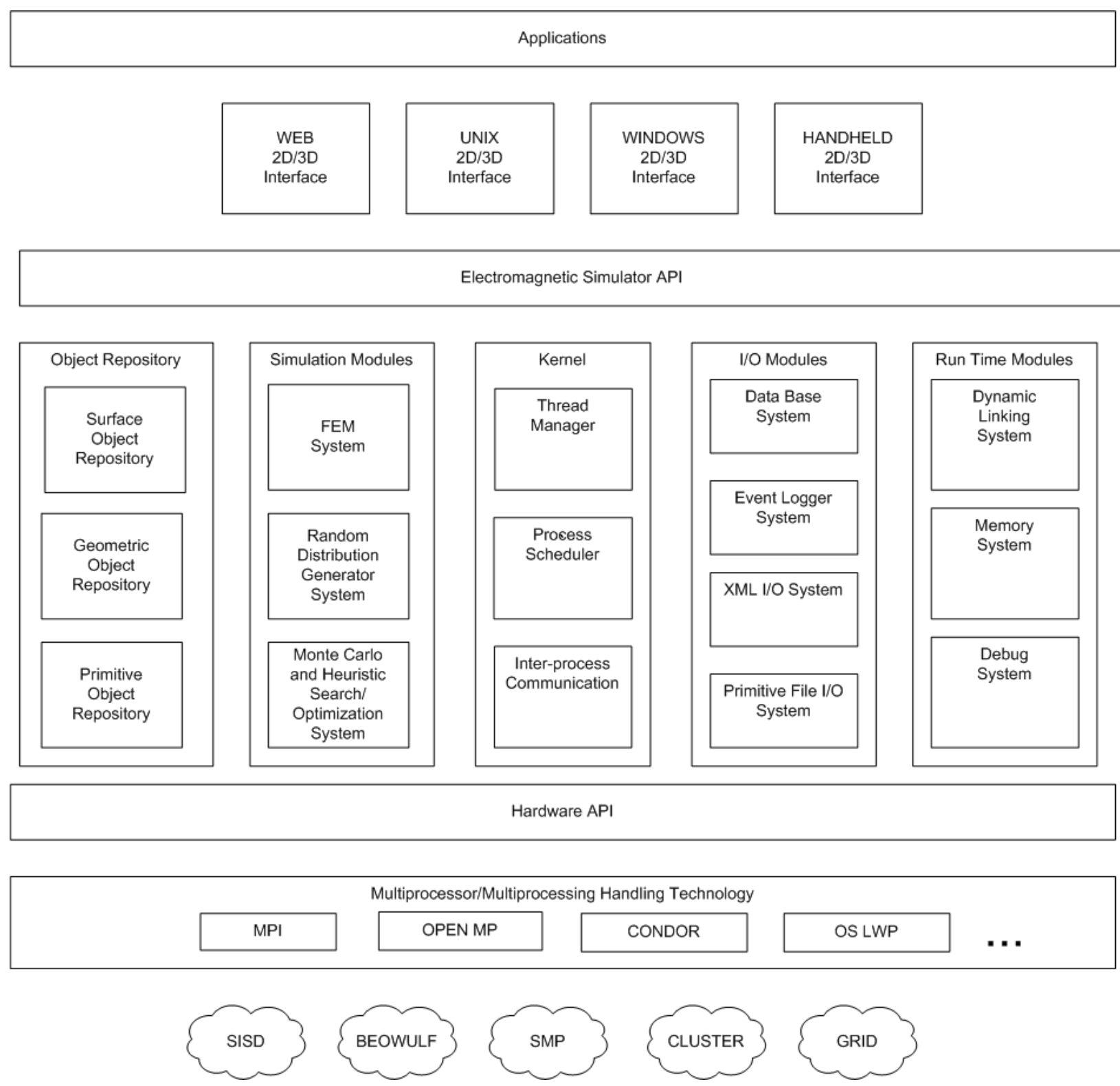

Figure 1. System architecture

Proceedings of the 2004 American Society for Engineering Education Annual Conference \& Exposition Copyright (C) 2004, American Society for Engineering 
An electromagnetic simulator API is created on top of the hardware API and has been divided into several modules: the object repository, simulation modules, kernel, input/output, and the running time routines. The object repository module consists mainly of a set of headers that encapsulate object oriented libraries that are publicly available to handle data structures and geometric representations. The simulation module contains the core routines for electromagnetic analysis including the implementation of the FEM system, optimization routines, and a generator of random sequences. The Kernel module implements the hardware API in order to execute different threads under systems with one or more processors. Within the I/O module are the routines to input data and to create logs and reports in a uniform way from/to a database management system using XML. Finally, the running time module consists of the implementation of routines required from the whole system during normal operations such as plug-in/dynamic-linkage, debugging, memory handling, and object creation/destruction routines.

Our plan is to make the system available under several platforms from the WEB to handhelds. For each of these platforms a common 2D/3D graphical user interface (GUI) will be developed with few variations in the implementation depending on the system.

Functionalities implementation: the Laplace equation solution algorithm

The purpose of this section is to illustrate the algorithm applied in the simulator to solve the Laplace equation for a given region of space using the FEM method. The development of the program basically involves four basic steps:

1. STEP I: Generate the mesh

1.1. Sketch the desired domain and enter the electric and geometric parameters

1.2. Enter the number of nodes and elements

1.3. Enter the fixed nodes

1.4. Generate the Nodal-Coordinate matrix containing the node global number and its coordinates

1.5. Generate the Fixed-Nodes containing the node global number and the correspondent potential

1.6. Generate the Global-to-Local mapping matrix containing the global element number and its local numbering

1.7. Generate the mesh

2. STEP II: Build the Global-Matrix

2.1. Evaluate the element coefficient matrices (Element Matrix)

2.2. Assemble the Global-Matrix

Proceedings of the 2004 American Society for Engineering Education Annual

Conference \& Exposition Copyright (C) 2004, American Society for Engineering 
2.2.1. Assemble the diagonal

2.2.2. Assemble the matrix upper block

3. STEP III: Evaluate the potentials for each free node

3.1. Assign an arbitrary initial value to the free nodes

3.2. Assemble the potential vector [V]

3.3. Apply the Iteration Method

4. STEP IV: Show the computation results

4.1. Tabular and graphical representation

4.2. Refine the results if necessary

Solving the Laplace equation: mesh generation

The field of finite element mesh generation encompasses numerous techniques. Finite Element Analysis (FEA) is an essential tool for the design and analysis of all engineering components and assemblies. FEA in its wider scope includes mathematical modeling, discretization, solution techniques and visualization. In the past, discretization was a manual labor-intensive task that took considerably longer than analysis. Today discretization is an automated process, which includes mesh generation and adaptive procedures. Adaptive refinement combined with a mesh generation technique permits the automatic specification of the mesh size to be used in the discretization.

Firstly it must make clear that the term "Mesh Generation" embodies several different tasks. The following subtasks can generally be identified: Initial Mesh, Re-meshing, and Refinement.

Mesh generation techniques: initial mesh generation

Initial or preliminary mesh generation requires a description of the geometry of the mesh domain. The mesh generator will then create a mesh of triangular elements that covers the domain according to the mesh size requirements specified by certain control parameters. In two dimensions the geometric description of the domain can be given as a series of node coordinates plus a description of the domain. In three dimensions a more complex boundary description is required.

Mesh generation techniques: re-meshing

The task of re-meshing is performed when a given mesh (generally referred to as the initial or coarse mesh) no longer satisfies criteria of mesh size or element quality. The mesh generator will extract the boundary definition of the initial mesh. Next the interior nodes and triangles will be discarded and the mesh generation task will continue in the same way as initial mesh generation. Alternatively rather than re-meshing the complete mesh as one domain the re-meshing may be 
carried out on an element by element basis as described below in the section "Element by element refinement".

Mesh generation techniques: refinement

Refinement occurs when additional elements are formed within an initial mesh. The refinement may be concentrated in a distinct area or areas. Refinement may be part of an adaptive procedure. Refinement of a mesh in a Delaunay triangulation context is defined as the addition of extra refinement nodes into the triangulation until mesh size criteria are satisfied. Depending on whether the initial mesh is a Delaunay triangulation or not, the initial mesh can be used immediately or the nodes have to be re-triangulated to form a valid Delaunay triangulation.

Mesh generation techniques: element by element refinement

The process of element by element refinement treats the initial coarse mesh as a series of independent meshes each of which are equivalent to just one coarse element. Each of the coarse elements is refined separately in the same way as the initial mesh generation task. Once all coarse elements are re-meshed, all new triangles are merged together to form the complete refined mesh domain.

Solving the Laplace equation: building the Global matrix

The first step in solving the Laplace equation is to obtain the first node of the first element from the Global-to-Local matrix. Then from the Nodal-Coordinate matrix the values of $x$ and $y$ corresponding to the node must be obtained. This process is repeated for all three nodes of the element. Knowing the $x$ and $y$ values of the nodes, the area of the element can be found using the FEM theory.

Once the area is known, the Element matrix for this triangle is straightforward. The procedure continues until half a matrix is known. The remaining part can be obtained applying the symmetry property that the matrix fulfills. The entire process ends when all the elements have been associated with the correspondent Element matrix.

After each element is characterized by its own Element matrix, the process to build the Global matrix $\mathrm{C}$ can be started. The Global matrix is built by scanning the Global-to-Local matrix. Depending upon a side or node coincidence between two elements, this Global matrix will be generated.

Suppose $\mathrm{G}(\mathrm{k}, \mathrm{i})$ is the value of the Global-to-Local matrix for the mesh element $k$ with local edge $i$ and $\mathrm{C}^{\mathrm{k}}$ is the Element matrix of the mesh element $k$. Each element $\mathrm{C}_{\mathrm{ij}}$ in the Global matrix is computed as the sum of the entries $C_{l m}^{k}$ so that $G(k, 1)=i$ and $G(k, m)=j$. Since the Global-toLocal matrix is symmetrical, the entries in $\mathrm{C}_{\mathrm{ij}}$ have to be computed only for $\mathrm{i} \leq \mathrm{j}$.

To facilitate the creation of this matrix, the diagonal and upper matrix triangle is generated separately. Since the Global matrix is symmetrical, the lower half of the matrix is also known. The dimensions of the Global matrix will be $\left[\mathrm{N}_{\mathrm{N}} \mathrm{x} \mathrm{N}_{\mathrm{N}}\right]$, where $\mathrm{N}_{\mathrm{N}}$ is the total number of nodes in the mesh.

Proceedings of the 2004 American Society for Engineering Education Annual Conference \& Exposition Copyright (C) 2004, American Society for Engineering 
Building the Global matrix: assembly of the diagonal

It is known that the values of the diagonal are always non-zero. Each value of the diagonal is given by the global node number contained within the Global-to-Local matrix. Each global number corresponds to a local number. The local number indicates the position in the current Element matrix from which the value is taken. Thus, if the $C_{k k}$ value is to be found, a scanning on the Global-to-Local matrix must be performed to find the global node number $\mathrm{k}$ associated with each single triangular element. If two or more single triangular elements share the same node, then their contributions must be added to form the specific value of the diagonal The Global matrix diagonal will have as many values as the number of nodes in the system.

Building the Global matrix: assembly of the upper block

To assemble to upper half of the Global matrix, a scan of the Global-to-Local matrix is also required. As mentioned before, each global number corresponds to a local number. The local number indicates the position in the current Element matrix from which the value is taken. If the $\mathrm{C}_{\mathrm{jk}}$ value is to be found, a scan is performed to determine those triangular elements which have global node numbers $j$ and $k$. This means that these two elements have a side in common. The contributions of these two elements are added in the $\mathrm{C}_{\mathrm{jk}}$ position of the Global matrix.

It must be pointed out that for a large number of nodes the Global matrix will result in a sparse matrix.

Solving the Laplace equation: the iteration process

To start the iteration process, set the potentials of fixed nodes to their known values and those of the free nodes to zero. With these values, the potentials at the free nodes are calculated and then used for the next iteration. The process is performed until the changes in the values between iterations are negligible.

The iteration process begins by setting the potentials of fixed nodes (where the potentials are known) to their prescribed values content into the Fixed-Nodes-Potential matrix generated during STEP I; then setting the potential at the free nodes (where the potentials are unknown) equal to zero. This first step has the Fixed-Nodes matrix as output.

With the values content into the Node-Potentials matrix the potentials at the free nodes are calculated using $\mathrm{V}_{\mathrm{k}}=-\frac{1}{\mathrm{C}_{\mathrm{kk}}} \sum_{\mathrm{i}=1, \mathrm{i} \neq \mathrm{k}}^{\mathrm{n}} \mathrm{V}_{\mathrm{i}} \mathrm{C}_{\mathrm{ki}}$. At the end of the first iteration, when the new values have been evaluated for all the free nodes they become the old values for the second iteration. This step has as output the new Node-Potentials matrix that will be used as input for the next iteration step.

The procedure is repeated until the change between subsequent iterations is sufficiently negligible or smaller than a prescribed value assigned at the beginning of the iteration process. 
Once the potentials at each node are calculated the potential distribution across the entire domain can be evaluated using $\mathrm{V} \times \mathrm{x}_{(,)} \mathrm{y}=\sum_{\mathrm{e}=1}^{\mathrm{N}_{\mathrm{E}}} \mathrm{V}_{\mathrm{e}} \mathrm{x}, \mathrm{y}$.

\section{Educational and Research Potential}

The launching of this project represents a new study topic for Engineering Technologists, and it provides the opportunity to learn about a subject that is not taught in the classroom. Research on the application of the Finite Elements Method (FEM) to electromagnetism, as well as the implementation of software routines needed for the analysis and simulation has initiated. As the project is developed, a deeper insight into structure analysis and simulation will come about.

The students involved have the opportunity to see how a project is generated starting from a simple idea. Teamwork and brainstorming are some of the techniques used to keep the process running. The Electromagnetic Structure Simulator exposes the students to a different workplace other than the classroom. It enhances their interaction and communication attempt to with other team members as well as improving their overall understanding of other subjects.

In the development of the project, the participants become familiar with the power of FEM as they analyze a specific domain. Furthermore, the exposure to this method enhances their abilities in other areas that come hand-in-hand with it. Engineering Technology students become involved in the development of programs and subroutines that must be implemented in order to obtain critical data. It is a global learning experience for students who do not have in-depth exposure to computational and algorithmic. In addition, the participants are afforded the opportunity to learn how to develop papers, pseudo codes and important documentation from a professional perspective.

\section{Current Status and Future Work}

This project has been structured in three main phases distributed over six semesters. Each main phase is divided into three sub-phases as listed below according to the activities in which each student is involved. At the time of writing this paper the project is in the first phase.

During the first two phases the simulation results will be compared with practical measurements performed by the microwave measurement kit and with results found in the relevant scientific literature. The third phase will require more sophisticated manufacturing techniques and measurement equipment; thus, the system output will be compared solely with the results found in the scientific literature.

Another important aspect included in this project is the "auto-training" of the students involved. From the first phase the top performing three students will be selected to serve as tutors for the newcomers. By the end of the third phase there will be a team capable of managing and developing new features for the system with the minimum support of faculty ${ }^{3}$.

- $\quad$ Phase 1

○ Design.

Proceedings of the 2004 American Society for Engineering Education Annual

Conference \& Exposition Copyright (C) 2004, American Society for Engineering

\section{Education}


- Definition of innovative EM models for basic passive isotropic structures.

- Design of an optimizing procedure using the Monte Carlo Method.

○ Implementation.

- Simulation and Comparison.

- Comparison of the system's simulation results with EM measurement performed using the same structures with microwave measurement kits.

- Comparison of the system's simulation results with EM measurement and simulations performed with the same structures and available in the scientific literature.

- $\quad$ Phase 2

○ Design.

○ Definition of innovative EM models for passive planar isotropic structures.

- Maintenance of the Monte Carlo optimizer. Definition of a new optimizing technique based on the Genetic Algorithm.

○ Implementation.

○ Simulation and Comparison.

O Comparison of the system's simulation results with EM measurement performed with the same structures using the microwave measurement kit.

- Comparison of the system's simulation results with EM measurement and simulations performed using the same structures and available in the scientific literature.

- $\quad$ Phase 3

○ Design.

○ Definition of innovative EM models for passive planar bi-isotropic multi-layers structures.

- Update the Monte Carlo and Genetic Algorithm optimizers and definition of a new optimizing technique based on the Taboo Search.

○ Implementation.

○ Simulation and Comparison.

Proceedings of the 2004 American Society for Engineering Education Annual Conference \& Exposition Copyright (C) 2004, American Society for Engineering 


\section{Conclusions}

In this paper has been presented the planning, development, current state and future work of an undergraduate research program intended to enhance engineering technology education at the University of Texas at Brownsville. The project, which is related to the simulation of electromagnetic waves, has been proven to be a successful tool to stimulate students to participate.

The ultimate goal is to institutionalize this program at UTB. At the end of each semester outgoing students will be asked to train incoming students to maintain and advance the development of the project. A database of outgoing students will be created in order to track their professional success in jobs or graduate school. Surveys and questionnaires will be applied at the end of the semester to obtain metrics and feedback. In this manner, is expected to sustain and improve the project and to continue to enhance the educational levels of Engineering Technology graduates.

\footnotetext{
${ }^{1}$ Finite element in electromagnetics for undergraduate curriculum

Obiozor, Clarence N. (Tuskegee Univ); Sadiku, Matthew N. O. Source: Conference Proceedings - IEEE SOUTHEASTCON, 1993, $4 \mathrm{p}$

Use of finite element electromagnetics packages in undergraduate courses

Ashen, R.A. (Infolytica Ltd); Colver, R.E.; Rashid, K. Source: COMPEL - The International Journal for Computation and Mathematics in Electrical and Electronic Engineering, v 14, n 4, Dec, 1995, p $327-331$

EMAP: A 3-D, finite element modeling code for analyzing time-varying electromagnetic fields

Hubing, Todd (Univ of Missouri-Rolla); Ali, Mohammad; Bhat, Girish Source: Applied Computational

Electromagnetics Society Journal, v 8, n 1, 1993, p 116-124

Electromagnetics CAD exercise for undergraduates

Sitzia, A. (Univ of Manchester) Source: International Journal of Electrical Engineering Education, v 29, n 4, Oct, 1992, p 291-296

Verification of transformer voltage with finite element method

Obiozor, Clarence N. (Univ of North Florida) Source: Conference Proceedings - IEEE SOUTHEASTCON, 1995, p 235-239

FEA of electromagnetics: A geometrical approach for problems with plane symmetry

Da Silva, J.A.P. (Applied Electromagnetics Laboratory, Escola Politecnica, Universidade de Sao Paulo); Rossi, Luiz Natal; Cardoso, Jose Roberto; Silva, Viviane Cristine; Lebensztajn, Luiz Source: IEEE Transactions on Magnetics, v 38, n 2 I, March, 2002, p 1313-1316

Teaching electromagnetic fields and FEM for undergraduate students

Lebensztajn, L. (Escola Polit. da Univ. de Sao Paulo); Silva, V.C.; Rossi, L.N.; Cardoso, J.R. Source: IEEE

Transactions on Education, v 44, n 2, May, 2001, p 209

LMGA-2D: A software package to teach FEA concepts

Abe, Nancy M. (Escola Politecnica da Universidade de Sao Paulo); Cardoso, Jose R.; Clabunde, Douglas R.F.;

Passaro, Angelo Source: IEEE Transactions on Magnetics, v 33, n 2 pt 2, Mar, 1997, p 1986-1989

Heitman, G. K., and Manseur, R., “Organization of a capstone design course," In 30th Annual Conference Frontiers in Education 2000, FIE 2000, Vol.1, pp. F1C/1-F1C/5, Oct 2000.

A. Leva, "A hands-on experimental laboratory for undergraduate courses in automatic control Education," IEEE Trans. Educ., vol. 46, pp. 263-272, May 2003.

F. Buret, D. Muller, and L. Nicolas, “Computer-aided education for magnetostatics,” IEEE Trans. Educ., vol. 42, pp. 45-49, Jan. 1999.

Burnett, David S. "Finite Element Analysis From Concepts to Applications,". Reading, Massachusetts: AddisonWesley, 1987.

C. S. Desai, and J. F. Abel, "Introduction to the finite element method: A numerical approach for engineering analysis," New York: Van Nostrand Reinhold, 1972.

${ }^{2}$ Matthew N. O. Sadiku "Numerical Techniques for Electromagnetics," CRC Pres John L.
} 
Volakis et al. 'Finite Element Method for Electromagnetics,' The IEEE/OUP Series on Electromagnetic Wave Theory Donald G. Dudley, Series Editor.

Tatsuo Itoh "Numerical Techniques for Microwave and Millimeter-wave Passive Structure," John Wiley \& Sons Editor.

${ }^{3}$ Y. Rahmat-Samii, and E. Michielssen, "Electromagnetic Optimization by Genetic Algorithms," John Wiley \& Sons Editor.

V. J. Rayward-Smith, I. H. Osman, C. R. Reeves (Editor), and G. D. Smith "Modern Heuristic Search Methods," John Wiley \& Sons Editor

J. B. Temes, "Teaching electromagnetic waves to electrical engineering students: an abridged approach," IEEE Trans. Educ., vol. 46, pp. 283-288, May 2003.

Baren, M.R.; Watson, J., "Developing communication skills in engineering classes," In Professional Communication Conference, 1993. IPCC 93 Proceedings. 'The New Face of Technical Communication: People, Processes, Products' , pp. 432-437, Oct. 1993.

FABIO URBANI has been a Visiting Associate Professor of Engineering Technology at UTB since 2002. Since then, he has taken an active role in developing improvements in the electronics engineering technology curriculum in the Department of Engineering Technology. From 1998 to 2002, Dr. Urbani was a senior consultant for several international telecommunications consultancy firms. He holds a Ph.D. in Electronics Engineering from the University of Rome La Sapienza, and a Bachelor of Electronics Engineering degree from the University of Rome La Sapienza.

JUAN R. IGLESIAS is Assistant Professor in the Computer Science Department at UTB. He obtained his Ph.D. in Computer Science from New Mexico State University. Dr. Iglesias' main fields of interest include artificial intelligence, databases, and algorithm analysis and design.

JOSE F. TREVIÑO is a senior student in Electrical Engineering Technology at the University of Texas at Brownsville. Throughout his engineering education, he has been exposed to many subjects including research, design, and programming projects. In addition, Mr. Treviño works as a math tutor for the University. This part-time job has given him a broad knowledge of mathematics and physics. He enjoys the engineering field and pretends to continue his education after graduation.

IRAM LEE is a senior student in Electrical Engineering Technology at the University of Texas at Brownsville. He has been involved in several microprocessor control related projects. In the spring of 2001, Mr. Lee was awarded with the Dean's List recognition. He is currently interested in pursuing a graduate degree in communication electronics. 